\title{
La pandemia producida por el virus SARS-CoV-2
}

\author{
The pandemic caused by the SARS-CoV-2 virus
}

\author{
Lucio D. Gómez-Melo ${ }^{a}$, Carlos A. González-Pérez ${ }^{b}$, Daniela León-Bernal ${ }^{c}$, José E. Ramírez- \\ Rodríguez, ${ }^{d}$ Mario I. Ortíz ${ }^{e}$
}

\begin{abstract}
:
With the appearance of SARS-CoV-2 the world has entered a state of emergency and has been quarantined since late 2019. SARSCoV-2 varies in incubation period, transmission rate, and lethality according to various indices in several countries. According to sequencing studies, it has been found to be a virus that mutated from an endemic strain of certain bats, which readily attaches to ACE-II receptors, which are expressed widely at the mucosal level of the respiratory system. Its main form of transmission is through respiratory droplets that are produced when we speak, cough or sneeze. Most cases are asymptomatic, which makes it difficult to diagnose and control the rate of infection. In those symptomatic cases a flu-like with general symptoms, pneumonia or acute respiratory distress syndrome may occur. For its diagnosis we can use CRP test, chest X-ray and in some cases CT scan as main tools. Currently there is no specific drug for the disease, but there are auxiliary and support treatments that have shown their effectiveness in some patients. A vaccine is being awaited.
\end{abstract}

Keywords:

SARS-CoV-2, COVID-19, Transmission, Diagnosis, Treatments

\begin{abstract}
Resumen:
Con la aparición de SARS-CoV-2 el mundo ha entrado en estado de emergencia y se ha puesto en cuarentena desde finales del 2019. SARS-CoV-2 varía su periodo de incubación, grado de contagio y letalidad de acuerdo a diversos índices de varios países. Según estudios de secuenciación se ha descubierto que es un virus que mutó de la cepa de un virus endémico de ciertos murciélagos, este se adhiere con facilidad a los receptores de ECA-II, los cuales se expresan de manera amplia a nivel de la mucosa del sistema respiratorio. Su principal forma de transmisión es a través de gotitas respiratorias que se producen cuando hablamos, tosemos o estornudamos. La mayoría de casos son asintomáticos, lo que dificulta el diagnóstico y el control de la tasa de contagios. En aquellos casos sintomáticos se puede presentar un cuadro gripal con síntomas generales, neumonía o síndrome de distrés respiratorio agudo, Para su diagnóstico nos podemos auxiliar de la prueba de PCR, radiografía torácica y en algunos casos TAC como principales herramientas. Actualmente no existe ningún fármaco específico para la enfermedad, pero existen tratamientos auxiliares y de soporte que han mostrado su eficacia en algunos pacientes. Se está a la espera de una vacuna.
\end{abstract}

Palabras Clave:

a Universidad Autónoma del Estado de Hidalgo, Instituto de Ciencias de la Salud, Pachuca, Hidalgo. México. https://orcid.org/00000003-4442-5628, Email: lucvio.dan@gmail.com

b Universidad Autónoma del Estado de Hidalgo, Instituto de Ciencias de la Salud, Pachuca, Hidalgo. México. https://orcid.org/00000002-3499-888X, Email: anderson.gz.cagp@gmail.com

c Universidad Autónoma del Estado de Hidalgo, Instituto de Ciencias de la Salud, Pachuca, Hidalgo. México. https://orcid.org/00000001-9857-9601, Email: daniibernal14@gmail.com

dUniversidad Autónoma del Estado de Hidalgo, Instituto de Ciencias de la Salud, Pachuca, Hidalgo. México. https://orcid.org/00000001-7234-9541, Email: jeduardorr52@gmail.com

e Autor de Correspondencia. Universidad Autónoma del Estado de Hidalgo, Instituto de Ciencias de la Salud, Pachuca, Hidalgo. México. https://orcid.org/0000-0003-1047-6304,Email: mortiz@uaeh.edu.mx 


\section{Introducción}

A finales del 2019 en Wuhan, China surgió un brote, al principio, identificado como una especie de casos de neumonía atípica cuya etiología era desconocida, y que posteriormente se identificó el agente causal como SARSCoV-2, que producía la enfermedad de COVID- $19^{1}$, el cual terminaría propagán dose a nivel mundial afectando así a miles de personas y a todos los países del mundo.

Actualmente, en lo que va del año, ha surgido un gran número de información sobre SARS-CoV-2, todo con el fin de poder entender su mecanismo fisiopatológico, la forma en la que se transmite, sus signos y síntomas, formas de poder detectarlo oportunamente y la búsqueda de un tratamiento eficaz para comenzar a controlar esta pandemia. Lamentablemente, no toda esta búsqueda de información ha tenido éxito, ya que aún se desconoce cuáles serían los signos y síntomas patognomónicos de COVID-192, cuál sería el tratamiento que muestre efectividad en la mayoría de los pacientes ${ }^{3}$, así como el estudio de los casos asintomáticos, que son de las principales fuentes de propagación ${ }^{4}$, el poder detectarlos y aislarlos oportunamente.

Como sabemos la enfermedad pasa por tres fases importantes ${ }^{3}$, en las cuales mientras más oportuno sea un diagnóstico, mejor expectativa hay sobre la evolución del paciente. Finalmente existen una serie de indicaciones para poder prevenir el contagio ${ }^{5}$, las cuales si se siguen al pie de la letra podrían llegar a reducir su propagación.

En el siguiente documento, se reúnen artículos, estudios, y opiniones recientes sobre el SARS-CoV-2, con el fin de poder brindar un panorama amplio sobre esta pandemia.

\section{Epidemiología}

El periodo de incubación de SARS-CoV-2 varía en promedio de acuerdo a múltiples estudios de 5.2 días a 12.5 días $^{6}$. Se han encontrado casos donde el periodo de incubación pasa los 19 días $^{7}$.

Los modelos de reproducción del virus al principio estiman que doblaban casos cada 7.4 días $^{6}$, este se calcula con base en la progresión clínica de la enfermedad, y al grado de contacto que tienen las personas. El ritmo básico de reproducción estimado, que es el número de casos nuevos que genera un caso a lo largo de su periodo infeccioso, por una gran mayoría de estudios es de 2.24 a $3.58 .^{8}$

Pero estos números han sido muy controversiales ya que dependiendo del país y los lugares de investigación el R0 (número reproductivo básico) del COVID-19 ha sufrido por grandes variantes siendo que en países poco desarrollados el número de casos nuevos que genera un caso es de 6.8 a 7 mientras que en países más desarrollados llegaba a ser de 2.4. ${ }^{2}$

Al inicio se consideraba que el COVID-19 tenía una letalidad muy baja, pero estos datos fueron cambiando porcada país ya que los factores de riesgo que predominan en cada país son diferentes y es que en México sus índices de letalidad están entre las tres más altas del mundo llegando a casi el $20 \%$, mientras que en países como España llegó hasta el $15 \%{ }^{9}$.

Los factores de riesgo de complicaciones incluyen edad avanzada (>65 años), enfermedad cardiovascular, enfermedad pulmonar crónica, diabetes, obesidad, hipertensión e inmunosupresión ${ }^{4}$.

\section{Virología}

Es importante que se identificara cuál fue el origen y la forma de transmisión de este virus, para poder desarrollar una forma de prevención para contener la infección.

En el 2019 el gobierno chino notificó al mundo sobre casos de neumonía de etiología desconocida, al hacer la investigaciones se encontró que el inicio del brote fue en un mercado de pescado de la ciudad de Wuhan, China, y de manera rápida se infectaron 50 personas ${ }^{1}$. Al estudiar el genoma de los infectados, se encontró un $79.5 \%$ de identidad entre SARS-CoV-2 y SARS-CoV y un $93,1 \%$ de identidad con la secuencia del Virus RaTG12 aislado de un murciélago ${ }^{10}$ lo que sugiere que SARS-CoV2 se originó de un virus endémico de ciertas especies de murciélago.

Existen cuatro principales subgrupos de coronavirus $(\alpha, \beta, \gamma, \delta)$. El SARS-CoV-2 pertenece al grupo $\beta$, las secuencias de aminoácidos dentro de los siete dominios conservados dentro del marco abierto de lectura genómica 1ab (ORF1ab) es $94.6 \%$ idéntico al original SARS-CoV. El virión del coronavirus mide 120$160 \mathrm{~nm}$ de diámetro, en su estructura tiene unas proyecciones, la proteína spike (S), lo que le confiere la clásica forma de corona. La proteína $S$ media la fijación y la fusión de membranas durante la infección. El coronavirus de igual forma posee tres proteínas estructurales adicionales, proteína de Membrana (M), 
proteína de Envoltura (E), Proteína de Nucleocápside $(\mathrm{N})^{10}$.

Un estudio comparativo de genomas encontró similitud con la Proteína S de un coronavirus aislado de un Pangolín, esto de igual forma sugiere que este mamífero podría ser el huésped intermediario entre el murciélago y el ser humano ${ }^{10}$, y en un estudio de fluorescencia se confirmó que SARS-CoV-2 usa el mismo receptor ECA-II y su mecanismo para entrar a la célula igual que lo hace SARS-CoV 1 .

\section{Fisiopatología}

La primera replicación ocurre en el epitelio de la mucosa de la parte superior del tracto respiratorio, con multiplicación adicional en el tracto respiratorio inferior y mucosa gastrointestinal, lo que ocasiona una viremia leve, que explica el por qué no genera síntomas de manera inmediata ${ }^{11}$.

Debido a que las proteínas de los viriones del Covid-19 tienen mayor afinidad hacia la ECA-ll y que esta se expresa de manera amplia en la mucosa nasal, bronquios, pulmones, corazón, esófago, riñón, estomago, vejiga e íleon, es capaz de provocar sintomatología diversa, pero de mayor gravedad en el aparato respiratorio ${ }^{11}$.

Una de las más grandes hallazgos que se han encontrado es la severa respuesta inflamatoria que ocurre durante la infección por SARS-Cov-2, lo que genera una inflamación descontrolada para nuestro sistema inmune pero no para el virus, ya que es a través de las disminución de la función reguladora de la ECA-II es responsable de la inflamación tan agresiva. EI SARS-Cov-2 se engancha a los receptores de entrada de las ECA-II lo que nos sugiere la probabilidad de que la misma cantidad de receptores de ECA-II que tenemos son los mismos que afecten al organismo, por la replicación de este virus, lo que generaría una replicación viral rápida que ocasiona una masiva muerte celular, epitelial y endotelial, junto con una importante fuga vascular, provocando la liberación excesiva de citocinas y quimiocinas proinflamatorias. Las células T-CD4 y CD8 muestran una hiperactivación en pacientes severos, la alta concentración de las células TCD4 proinflamatorias y los gránulos citotóxicos de las células T-CD8 sugieren una respuesta inmunitaria antiviral y una excesiva sobre activación de las células $\mathrm{T}$, lo que sugiere un factor crítico para sintomatología severa 0 incluso la muerte ${ }^{11}$.

Los hallazgos patológicos que podemos encontrar en esta enfermedad, se da en aquellas personas que tiene un cuadro severo, ya que podemos encontrar un daño difusos bilateral y en un exudado muestra fibrosis, estos alvéolos pulmonares muestran una evidente descamación de neumocitos y de su membrana hialina, lo que nos indica un síndrome de dificultad respiratorio. También se han encontrado casos en donde el tejido pulmonar izquierdo, presenta edema con formación de membrana hialina, lo que nos sugiere un síndrome de dificultad respiratoria aguda en fase temprana, se llega a presentar infiltrados inflamatorios, ocasionados por linfocitos, ambos pulmones presentan cambios de tipo citopático en las etapas tardías y más graves de la enfermedad. ${ }^{[2]}$

EI SDRA es una afección pulmonar potencialmente mortal que impide que se del correcto intercambio de gases entre los pulmones y la circulación, lo que genera menor captación de oxígeno, explicando la principal causa de muerte ${ }^{11}$.

\section{Modo de transmisión}

EI SARS-CoV-2 se transmite principalmente de personapersona a través de gotitas respiratorias, que generalmente se liberan cuando una persona infectada tose o estornuda. Debido a que las gotas generalmente caen dentro de unos pocos metros, la probabilidad de transmisión aumenta si las personas permanecen cercanas la una a la otra menos de 2 metros, a este modo de transmisión se le conoce como contacto directo.

La transmisión por contacto indirecto se produce cuando hay contacto con superficies $u$ objetos contaminados 4 .

Un estudio reportó que se aisló SARS-CoV-2 de las heces fecales de un paciente con una neumonía severa el 10 de febrero del 2020 en Guangdong, China ${ }^{12}$. Mientras que otro estudio aisló SARS-CoV-2 en hisopos fecales, en sangre y en orina ${ }^{10,13}$, lo cual sugiere la probabilidad de múltiples rutas de transmisión.

La proteína ECA-II presente en abundancia en las célu las del epitelio alveolar de los pulmones, así como en los enterocitos del intestino delgado ${ }^{14}$ nos puede ayudar a entender las rutas de infección y las manifestaciones de la enfermedad ${ }^{13}$.

De igual forma se plantea la probabilidad de una transmisión Materno-Fetal, puesto que el 6 de febrero de 2020, el hospital Wuhan Tongji en China reportó el caso de una mujer embarazada infectada con SARS-CoV-2 que dio a luz a un niño que mostró resultados positivos a SARS-CoV-2 a las 36 horas del parto. Mientras que 2 días después, el 8 de febrero del mismo año, en Zhejiang, China, una mujer con una severa infección de SARS-CoV2 dio a luz a un recién nacido que dio negativo a muchas pruebas para SARS-CoV-2, esto puede sujetar la posibilidad de una transmisión Materno-Fetal a una simple teoría ${ }^{10}$, Sin embargo un estudio reciente reveló que la infección fetal puede ser transmitida al final del 
embarazo ${ }^{15}$, esto debido a la baja expresión de ECA-\| entre las células detectadas en la interfase Materno-Fetal (Placenta).

\section{Manifestaciones Clínicas}

El $97.5 \%$ de los pacientes sintomáticos presentarán síntomas dentro de los 11.5 días posteriores a la infección.

- Etapa I (Fase leve): El virus se multiplica a tal grado que coloniza el tracto respiratorio del hospedero, causando un cuadro gripal, con malestar general, fiebre y tos.

- $\quad$ Etapa II (Fase moderada): Es caracterizada por el desarrollo de neumonía, esta puede ser leve, moderada o grave ${ }^{3}$.

Es importante tener conocimiento de que algunos pacientes pueden presentar síntomas gastrointestinales, como anorexia, náuseas y diarrea. También se ha informado de anosmia y ageu sia ${ }^{4}$.

- Etapa III (Fase grave): Se manifiesta como un síndrome de inflamación sistémica extrapulmonar. En esta etapa son evidentes el shock, la insuficiencia respiratoria, síndrome de disfunción multiorgánica e incluso colapso cardiopulmonar ${ }^{3}$.

Los indicadores de enfermedad grave son taquipnea marcada (>30 respiraciones por minuto), hipoxemia (saturación de oxígeno <93\%) e infiltrados pulmonares. Los pacientes con COVID-19 grave tienen un riesgo elevado de enfermedad crítica prolongada y muerte ${ }^{11}$.

\section{Estudios Diagnósticos}

El diagnóstico es parte importante del armamento en la lucha contra el COVID-19, el primer método diagnóstico es la prueba de RT-PCR el cual detecta la secuencia viral, la Administración de Drogas y Comida China aprobó un lote de kit cuantitativos que detectan la secuenciación por fluorescencia que permite el diagnóstico pero tenía un gran problema al dar muchos casos de falsos negativos y solucionaron el problema, empezaron a utilizar el ácido nucleico como prueba diagnóstica de mayor eficacia ${ }^{11}$.

También se ha empleado la prueba serológica en combinación con inmunocromatográfica y otras técnicas han resultado ser más efectiva y con mayor rapide $z^{11}$. La radiografía de tórax es de las herramientas más importantes que tenemos para un buen diagnóstico para el COVID-19 en práctica clínica, ya que la mayoría de casos tienen características similares, las cuales incluyen: opacidad de vidrio pulido en distribución bilateral, como la más representativa de la enfermedad, pero no debe de ser utilizada como primer prueba de diagnóstico ya que pacientes que apenas empiezan a cursar con una sintomatología aparecen sin anormalidades, las radiografías de tórax están indicados en aquellos pacientes cuyos síntomas han perdurado por más de 1 semana y son casos altamente sospechosos ${ }^{11,17}$.

\section{Manejo}

Actualmente no hay un agente antiviral específico aprobado para COVID-19. Las bases para el manejo de soporte incluyen mantenimiento de vía aérea permeable, oxigenoterapia y manejo de líquidos. Las estrategias terapéuticas deben elegirse según la etapa de la infección. En caso de encontrarse en etapa I, el tratamiento es sintomático se recuperan en su hogary en aislamiento. De encontrarse en etapa II amerita ingreso para observación y tratamiento, el cual consiste principalmente en medidas de soporte respiratorio.

De acuerdo a Marín et al., 2020 el efecto antiinflamatorio de los corticosteroides ayu daría mejorar la evolución clínica de estos pacientes. Sin embargo, hasta el momento no hay evidencia suficiente para recomendar el tratamiento con corticosteroides en pacientes con COVID-19.

Cuando se presenta la etapa III se propone el uso de inmunomoduladores, con el fin de disminuir la aceleración inmunológica que la caracteriza. Sin embargo, también se ha llegado a sugerir el uso de corticosteroides e inhibidores de las interleucinas 1 y $6^{3}$.

Dado el alto riesgo de diseminación nosocomial, se necesitan procedimientos estrictos de control de infecciones.

Los pacientes deben ser monitoreados cuidadosamente por observación clínica y oximetría de pulso. Decidir si intubar o no, es un aspecto crítico del cuidado de pacientes gravemente enfermos con COVID19.

Posibles indicaciones clínicas para la intubación endotraqueal $^{16 .}$ :

- Obstrucción inminente de la vía aérea

- Señales de respiración inestable

- Hipoxemia refractaria

- Hipercapnia o acidemia

- Encefalopatía o protección inadecuada de vía aérea

No se recomienda el uso de los siguientes medicamentos: Oseltamivir, Ivermectina, Azitromicina, Nitaxozanida, Factor de transferencia, Cloroquina e Hidroxicloroquina, Colchicina, Antioxidantes, Nanomoléculas de cítricos, Dióxido de cloro, Inmunoglobulina intravenosa e Interferones

Algunos de los fármacos que se están utilizando en protocolos de investigación son: Remdesivir, 
Lopinavir/Ritonavir, Baricitinib, Tocilizumab, Anakinra, Plasma convaleciente y Heparinas a diversas dosis ${ }^{3}$.

De acuerdo a un estudio realizado en la Universidad Pública de Marseille, Francia, se está investigando el uso de Teicoplanin el cual es un antibiótico glucopéptido que se usa para tratar infecciones bacterianas, pero resultó ser funcional ante SARS-CoV y se cree puede igual serútil ante SARS-CoV-2. Teicoplanin ha resultado ser eficaz en el tratamiento de algunos virus tales como Ébola, Influenza, Flavivirus, Hepatitis C y VIH, así como MERS y SARS-CoV. De acuerdo con el estudio, Teicoplanin actúa en una etapa temprana del ciclo viral inhibiendo la hendidura de $\mathrm{pH}$ bajo de la proteína viral $\mathrm{S}$, previniendo así la liberación del genoma ARN viral frenando el ciclo de replicación. Lo que el estudio demuestra es que esta actividad de igual forma se conserva en SARS-CoV-2 ${ }^{[18]}$, estos resultados preliminares ahora están por confirmarse en un estudio clínico aleatorizado

\section{Medidas generales de prevención para población en general}

Existen medidas establecidas para poder frenar la propagación y evitar así el contagio de COVID-19[5]

- Lavado frecuente de manos con agua y jabón al menos por 60 segundos.

- En el caso de que las manos no estén visiblemente sucias o contaminadas, se puede utilizar un producto para desinfección de manos que contenga $70 \%$ de alcohol (por 20-30 segundos).

- Limpiar y desinfectar los objetos y las superficies que se tocan con frecuencia.

- Cubrirse la nariz y la boca al toser o estornudar.

- Evitar el contacto cercano con personas que padecen infecciones respiratorias agudas (mantenerse al menos metro y medio de distancia).

- Evitar estar en lugares muy concurridos.

- Evitar tocarse la cara.

- Evitar saludar de mano y beso.

\section{Control y prevención de infecciones}

Los trabajadores de la salud deben estar protegidos contra la infección por SARS-CoV-2, por lo que deben usar equipo de protección personal. EI EPP debe incluir como mínimo, bata de aislamiento, guantes, cubrebocas y protección para los ojos, ya sean gafas o careta.

\section{Procedimiento para ponerse el EPP}

Antes de ponerse el EPP:
- Asegurarse de estar bien hidratado y usar el baño, ya que se puede llegar a usar el EPP por un tiempo prolongado que no permite realizar estas acciones.

- Asegurar el cabello largo y retirar objetos personales como joyas

- Lavarse las manos con agua y jabón

- Verificar la integridad del equipo

- Cuando se use el respirador N95:

- Colocar la parte externa del respirador con la mano dominante, sosteniendo la tira flexible con las yemas de los dedosy dejando que las correas cuelguen libremente alrededor de la mano

- Colocar el respirador en la cara

- Asegurar la correa inferior alrededor de la parte superior del cuello y asegurar la correa superior detrás de la cabeza

- Moldear la tira nasal flexible alrededor del puente nasal con ambas manos

- Colocar el protector facial

- Luego de colocar el respirador:

- Colocar guantes y extender los puños sobre las mangas de la bata

\section{Procedimiento para quitar el EPP}

- Quitarse un guante con la otra mano

- Agarrar el exterior del guante por la muñeca, quitando el guante de la mano con el exterior contaminado doblado hacia adentro

- Sostener el guante retirado en la mano enguantada

- Deslizar un dedo debajo de la pulsera del guante restante

- Suavemente quitarse el guante que queda, formando una bolsa para el otro guante

- Desecharlo y desinfectarse las manos

- Quitarse la banda desabrochando el cierre de la cintura

- Sujetar el área de los hombros y retirar la bata, volteándola hacia adentro y envolviéndola en un paquete

- Desechar la bata y desinfectarse las manos

- Retirar la careta levantándola por encima de la cabeza sin tocar el escudo

- Retirar el respirador N95, tocando solo las correas y desinfectar las manos ${ }^{[19]}$

\section{Conclusión}

Como sabemos la infección porSARS-CoV-2, au menta en su propagación día con día, su principal fuente de contagio son las partículas que producimos al hablar, toser y 
estornudar, así como el contacto entre superficies contaminadas con las manos y después llevarlas a ojos, boca o nariz. Ya que COVID-19 no tiene una sintomatología específica, es importante acudir a un centro de salud ante la primera sospecha de infección. A la fecha no se cuenta con un tratamiento efectivo y es por eso que lo mejor que se puede hacer para prevenir su propagación es seguir las medidas generales de prevención para población en general.

\section{Referencias}

[1] Shereen, M. A., Khan, S., Kazmi, A., Bashir, N., \& Siddique, R. (2020). COVID-19 infection: Origin, transmission, and characteristics of human coronaviruses. Journal of advanced research, 24, 91-98. https://doi.org/10.1016/j.jare.2020.03.005

[2] Trilla, A. (2020). Un mundo, una salud: la epidemia por el nuevo coronavirus COVID-19: Medicina clínica. ELSEVIER, 154(5), 175177. https://doi.org/10.1016/j.medcli.2020.02.002

[3] Oliva Marín, J. (2020). Posibles estrategias de tratamiento para COVID-19. Alerta, Revista Científica Del Instituto NacionalDe Salud, 3(2). https://doi.org/10.5377/alerta.v3i2.9626

[4] Gandhi, R., Lynch, J., \& del Río, C. (2020, 24 abril). Mild or moderate Covid-19. The New England Journal of Medicine. https ://www.nejm.org/doi/pdf/10.1056/NEJMcp2009249

[5] Aragón-Nogales, R., Vargas-Almanza, I., \& Miranda-Novales, M. G. (2020, 2 marzo). COVID-19 por SARS-CoV-2: la nueva emergencia de salud. Revista Mexicana de Pediatría. https://www.medigraphic.com/pdfs/pediat/sp-2019/sp196a.pdf

[6] Zhai, P., Ding, Y., Wu, X., Long, J., Zhong, Y., \& Li, Y. (2020). The epidemiology, diagnosis and treatment of COVID-19. International journal of antimicrobial agents, 55(5), 105955. https://doi.org/10.1016/j.ijantimicag.2020.105955

[7] Bai, Y., Yao, L., Wei, T., Tian, F., Jin, D. Y., Chen, L., \& Wang, M. (2020). Presumed Asymptomatic Carrier Transmission of COVID-19. JAMA, 323(14), 1406-1407. Advance online publication. https://doi.org/10.1001/jama.2020.256

[8] Lai, C. C., Shih, T. P., Ko, W. C., Tang, H. J., \& Hsueh, P. R. (2020). Severe acute respiratory syndrome coronavirus 2 (SARS-CoV-2) and coronavirus disease-2019 (COVID-19): The epidemic and the challenges. International journal of antimicrobial agents, 55(3), 105924. https://doi.org/10.1016/j.ijantimicag.2020.105924

[9] Suárez V, et al. Epidemiología de COVID-19 en México: del 27 de febrero al 30 de abril de 2020. Rev Clin Esp. 2020. https://doi.org/10.1016/j.rce.2020.05.007

[10] Shi Y, Wang G, CaiXP, et al. An overview of COVID-19. J Zhejiang Univ Sci B. 2020;21(5):343-360. doi:10.1631/jzus.B2000083

[11] Jin, Y., Yang, H., Ji, W., Wu, W., Chen, S., Zhang W., \& Duan, G. (2020). Virology, epidemiology, pathogenesis, and control of COVID$19 ; 12(375), 10.3390 / \mathrm{v} 12040372$

[12] Guo, Y. R., Cao, Q. D., Hong, Z. S., Tan, Y. Y., Chen, S. D., Jin, H. J., Tan, K. S., Wang, D. Y., \& Yan, Y. (2020). The origin, transmission and clinical therapies on coronavirus disease 2019 (COVID-19) outbreak - an update on the status. Military Medical Research, 7(1), 11. https://doi.org/10.1186/s40779-020-00240-0

[13]Zhang, W., Du, R. H., Li, B., Zheng, X. S., Yang, X. L., Hu, B., Wang, Y. Y., Xiao, G. F., Yan, B., Shi, Z. L., \& Zhou, P. (2020). Molecular and serological investigation of 2019-nCoV infected patients: implication of multiple shedding routes. Emerging microbes \& infections, 9(1), 386-389. https://doi.org/10.1080/22221751.2020.1729071

[14]Hamming, I., Timens, W., Bulthuis, M. L., Lely, A. T., Navis, G., \& van Goor, H. (2004). Tissue distribution of ACE2 protein, the functional receptor for SARS coronavirus. A first step in understanding SARS pathogenesis. The Journal of pathology, 203(2), 631-637. https://doi.org/10.1002/path.1570

[15]Chen, H., Guo, J., Wang, C., Luo, F., Yu, X., Zhang, W., Li, J., Zhao, D., Xu, D., Gong, Q., Liao, J., Yang, H., Hou, W., \& Zhang, Y. (2020). Clinical characteristics and intrauterine vertical transmission potential of COVID-19 infection in nine pregnant women: a retrospective review of medical records. Lancet (London, England), 395(10226), 809-815. https://doi.org/10.1016/S0140-6736(20)30360-3

[16] Berlin, D. A., Gulick, R. M., \& Martinez, F. J. (2020, 15 mayo). Severe Covid-19. The New England Journal of Medicine. https://www.nejm.org/doi/pdf/10.1056/NEJMcp2009575

[17]Paez_Granada, D., Chicaiza, M., Saritama, G., Ávalos, C., Cabezas, H., \& Andrade. J. (2020). Diagnóstico de pacientes con sospecha de COVID-1: ¿Cuál es el rol de la Tc de Tórax?; 155(2): 93. 10.1016/j.medcli.2020.04.001

[18]Baron, S. A., Devaux, C., Colson, P., Raoult, D., \& Rolain, J. M. (2020). Teicoplanin: an alternative drug for the treatment of COVID19?. International journal of antimicrobial agents, 55(4), 105944. https://doi.org/10.1016/j.ijantimicag.2020.105944

[19] Ortega, R., Gonzalez, M., Nozari, A., \& Canelli, R. (2020, 19 mayo). Personal Protective Equipment and Covid-19. The New England Journal of Medicine. https://www.nejm.org/doi/pdf/10.1056/NEJMvcm2014809?articleToo ls=true 